\title{
A Short Review of Relativistic Iron Lines from Stellar-Mass Black Holes
}

\author{
J. M. Miller ${ }^{1}$ \\ University of Michigan Department of Astrophysics, 500 Church Street, Ann Arbor MI 48109-1042 USA, \\ jonmm@umich.edu \\ Received 01 Sept 2006, accepted 11 Oct 2006 \\ Published online later \\ Key words X-rays: binaries - Accretion, accretion disks \\ In this contribution, I briefly review recent progress in detecting and measuring the properties of relativistic iron lines \\ observed in stellar-mass black hole systems, and the aspects of these lines that are most relevant to studies of similar lines \\ in Seyfert-1 AGN. In particular, the lines observed in stellar-mass black holes are not complicated by complex low-energy \\ absorption or partial-covering of the central engine, and strong lines are largely independent of the model used to fit the \\ underlying broad-band continuum flux. Indeed, relativistic iron lines are the most robust diagnostic of black hole spin that \\ is presently available to observers, with specific advantages over the systematics-plagued disk continuum. If accretion \\ onto stellar-mass black holes simply scales with mass, then the widespread nature of lines in stellar-mass black holes may \\ indicate that lines should be common in Seyfert-1 AGN, though perhaps harder to detect.
}

(c) 2006 WILEY-VCH Verlag GmbH \& Co. KGaA, Weinheim

\section{Introduction}

Broad Fe $\mathrm{K}$ emission lines plausibly arising in the inner disk around black holes, and shaped by the strong Doppler shifts and gravitational redshifts expected there, were first reported in stellar-mass black holes (Barr, White, \& Page 1985; van der Woerd, White, \& Kahn 1989). Though these detections were achieved with low-resolution gas spectrometers, their discovery was met with enthusiasm, and theoretical papers showing their reported widths to be consistent with extreme dynamical broadening soon followed (Fabian et al. 1989, Laor 1991). It was also quickly realized that Fe K emission lines are merely the most prominent feature of the overall response of an accretion disk to external irradiation by hard X-ray flux (George \& Fabian 1991).

Although strong Fe K lines in Seyfert AGN were wellknown at the time broad lines were found in stellar-mass black holes, the lower flux in AGN lines made it difficult to measure their shape. In the ASCA era, however, studies of broad iron lines in Seyfert-1 AGN advanced rapidly. The SIS (solid-state imaging spectrometer, an X-ray CCD spectrometer) aboard ASCA revealed that the lines in some Seyfert-1 AGN were not only broad, but asymmetric consistent with strong Doppler shifts and gravitational redshifts expected in the inner accretion disk (see, e.g. Tanaka et al. 1995; Iwasawa et al. 1996; Nandra et al. 1997a, Nandra et al. 1996b). Although the SIS was well-suited to studies of the line profile in Seyfert-1 AGN, ruinous photon pileup resulted when stellar-mass black holes were observed, greatly complicating efforts to recover robust spectra for detailed line measurements (see, e.g., Ebisawa et al. 1996).

In the XMM-Newton and Chandra era, considerable effort has again been devoted to the study of relativistic iron lines. While relativistic lines appear to be confirmed in some cases, complex low-energy absorption or alternative continuum spectral models initially cast some doubt on relativistic lines in other cases (see, however, the contributions by Fabian et al. Nandra et al. and Reeves et al. in these proceedings). In the stellar-mass black hole regime, the fast read-out modes and advanced spectrometers aboard XMMNewton and Chandra have made it possible to measure the properties of broad iron lines without photon pile-up. A number of asymmetric lines have been revealed, showing that they are also shaped by relativistic blurring at the inner accretion disk. In this sense, XMM-Newton and Chan$d r a$ have done for stellar-mass black holes what ASCA did for Seyfert-1 AGN.

In Section 2, recent observations of relativistic $\mathrm{Fe} \mathrm{K}$ lines in stellar-mass black holes are reviewed. In Section 3 , the robustness of relativistic Fe $\mathrm{K}$ lines and their utility as spin diagnostics is critically examined. In Section 4, different methods of constraining black hole spin are discussed. Finally, the relevance of relativistic lines in stellarmass black holes to studies of Seyfert-1 AGN and objectives for the future studies of relativistic lines are discussed in Section 5.

\section{Recent Observations of Relativistic Lines}

The first clear detection of a relativistic $\mathrm{Fe} \mathrm{K}$ emission line at moderate or high resolution was made in a Chandra High Energy Transmission Grating Spectrometer (HETGS) observation of Cygnus X-1 (Miller et al. 2002a). The resolution of the HETGS permitted a narrow iron line, either due to illumination of the companion wind or outer disk, to be separated from an underlying broad, red-shifted emission 


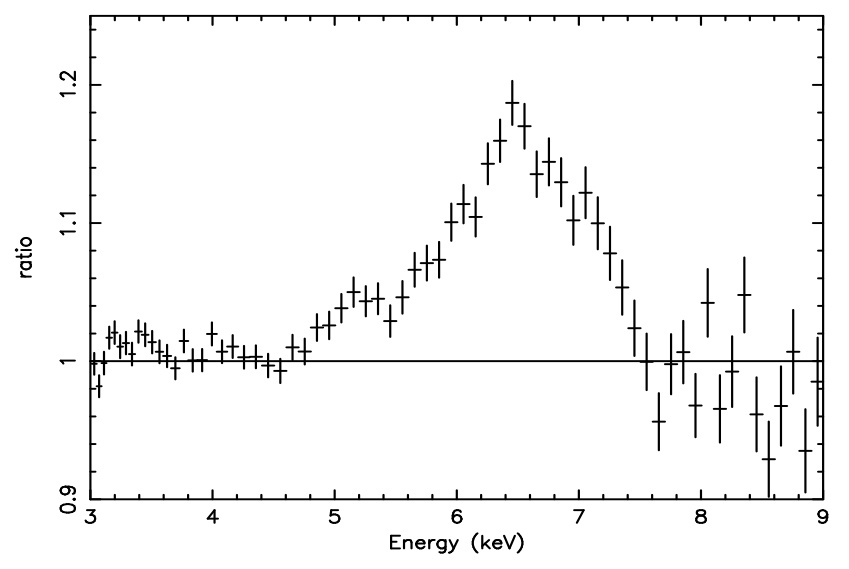

Fig. 1 The data/model ratio above shows the relativistic Fe K emission line profile revealed after fitting a phenomenological model to a recent $X M M-N e w t o n$ observation of Cygnus X-1 in a high flux state (Miller et al. 2006, in prep.). Fits with relativistic line models indicate that Cygnus X-1 may harbor a black hole with low or moderate spin, rather than high spin like many black holes with much older companion stars. This suggests that accretion may be more important than black hole formation events in driving spin parameters.

line. The observed line profile does not require a high degree of black hole spin. Cygnus X-1 was in an "intermediate" flux state when it was observed with Chandra; at higher flux levels, stronger line emission is expected, and more sensitive spectra can be obtained. More recent and more sensitive XMM-Newton/EPIC-pn observations of Cygnus X-1 confirm the broad line profile at CCD resolution (see Figure 1; Miller et al. 2006, in prep).

Soon after the Chandra/HETGS observation of Cygnus X-1, a new black hole transient - XTE J1650-500 - was observed with the XMM-Newton/EPIC-pn camera. This observation revealed a strongly skewed Fe $\mathrm{K}$ emission line profile, and fits with the Laor line model imply that the disk extends to within $2 G M / c^{2}$, requiring a black hole spin parameter in excess of $a \simeq 0.9$ (Miller et al. 2002b). Importantly, the line profile and overall disk reflection spectrum is well fitted with a model that is remarkably similar to that which describes the same components in an XMM-Newton observation of the Seyfert-1 galaxy MCG-6-30-15 (Fabian et al. 2001).

The relativistic line in XTE J1650-500 was confirmed in observations made by BeppoSAX, and the observed line variability is consistent with the predictions of a variability model based on gravitational light bending near to a spinning black hole (Miniutti, Fabian, \& Miller 2004). The same pattern of variability was later reported in an analysis of RXTE spectra (Rossi et al. 2005). It is again important to note that this pattern of variability has also been observed in the Seyfert-1 galaxies (MCG-6-30-15: Miniutti et al. 2003; 1H 0419-577: Fabian et al. 2005; NGC 4051: Ponti et al. 2006).

An XMM-Newton observation of the Galactic black hole candidate SAX J1711.6-3808 revealed another broad Fe K emission line (in 't Zand et al. 2002a). The analysis of the $X M M-N e w t o n$ data was complicated by photon pile-up, but the line is confirmed in simultaneous BeppoSAX spectra. The line profile is more symmetric than many others, but is consistent with being shaped by Doppler shifts and gravitational red-shifts at the inner accretion disk.

GX 339-4 has been a particularly important source for recent studies of relativistic iron lines in stellar-mass black holes. As with Cygnus X-1, a Chandra/HETGS observation made in an intermediate flux state first clearly revealed a relativistic line in this source (Miller et al. 2004a). Fits to the Chandra spectrum with a relativistic line and disk reflection model suggest that the disk extended to within $3 G M / c^{2}$, again requiring a high black hole spin parameter (Miller et al. 2004b). A long observation made with the $X M M-N e w t o n / E P I c-p n$ camera in a higher flux state discovered the most skewed Fe $\mathrm{K}$ emission line yet found in a stellar-mass black hole: from a centroid energy consistent with Fe XXV (6.70 keV) and/or Fe XXVI (6.97 keV), the red wing extends down to approximately $3 \mathrm{keV}$, requiring $r \leq 2 G M / c^{2}$ or $a \geq 0.9$. Again, the line profile and the overall continuum spectrum are remarkably similar to the most extreme line profiles detected in MCG-6-30-15 (e.g. Fabian et al. 2001), and the spectrum can be fit with very similar models. Most recently, a very long observation of GX 339-4 in a low flux state revealed a relativistic line at a low mass accretion rate, suggesting that the disk remains close to the black hole even at low accretion rates (Miller et al. 2006a).

Strong Fe K emission lines are not frequently observed in soft spectral states, consistent with the expectation that a strong external source of hard X-rays is needed to irradiate the disk. Even in some hard phases, however, lines can appear to be weak, and/or too narrow to be produced in a disk that extends very close to the black hole. An XMMNewton observation of the "microquasar" GRS 1915+105 revealed a broad line that is inconsistent with an origin in a disk close to the black hole in this system (Martocchia et al. 2006). This line shape is inconsistent with profiles observed previously with BeppoSAX when the source was in different states. However, in other phases, the line profile in GRS $1915+105$ is consistent with originating close to the black hole (Martocchia et al. 2002, Miller et al. 2004c).

Most recently, a skewed Fe $\mathrm{K}$ line was detected in long XMM-Newton observations of GRO J1655-40 (Diaz Trigo et al. 2006). The source was observed in a high flux state, similar to the phase in which an extremely skewed line was found in GX 339-4. GRO J1655-40 is an extraordinary system in which independent evidence of black hole spin has been reported, e.g. from high frequency quasi- periodic oscillations (QPOs; see Strohmayer 2001). The line profile revealed with XMM-Newton also signals a black hole with a high spin parameter: fits with a relativistic line model again signal an inner disk radius within $2 G M / c^{2}$, corresponding to $a \geq 0.9$. 
In general, $\mathrm{Fe} \mathrm{K}$ emission lines have been revealed in deep exposures of black holes in sufficiently bright and hard spectral states, made with a moderate (CCD) or high (gratings) resolution spectrometers. However, exceptions exist among highly obscured and high-inclination systems. Observations of the black hole candidates $4 \mathrm{U} 1630-472$ and H 1743-322 have not revealed broad Fe K emission lines, though blue-shifted $\mathrm{Fe}$ absorption lines have been discovered (Miller et al. 2006b).

The success of XMM-Newton and Chandra observations of relativistic Fe K lines in stellar mass black holes bolsters detections claimed in spectra obtained with low resolution gas spectrometers. It has also motivated both new observations and archival analysis with gas spectrometer data. Apart from the line in GRS 1915+105 and SAX J1711.63808 discussed above (Martocchia et al. 2002; in 't Zand et al. 2002), BeppoSAX also observed broad Fe $\mathrm{K}$ lines in Cygnus X-1 (Frontera), XTE J1908+094 (in 't Zand 2002b), and V4641 Sgr (Miller et al. 2002c). An analysis of archival ASCA gas imaging spectrometer (GIS) data revealed detections of broad, relativistic lines in XTE J1550-564, GRO J1655-40, GRS 1915+105, and Cygnus X-1 (Miller et al. $2004 \mathrm{c}$ ). Observations with RXTE, though it is not a focusing telescope, have detected broad Fe $\mathrm{K}$ lines in all of the systems mentioned above, and many others. RXTE has been particularly important to the detection of broad $\mathrm{Fe} \mathrm{K}$ lines in black holes, as it is extremely well suited to defining the broad-band X-ray continuum in the 3-200 keV band.

\section{On the Robustness of Fe K Lines}

Studies of relativistic lines in Seyfert-1 galaxies have some specific advantages over comparable studies in stellar-mass black holes. The massive black holes in Seyfert galaxies allow one to study dynamical timescales that are inaccessible in stellar-mass black holes, providing an improved view of the innermost region near to the black hole. Galactic stellarmass black holes, however, are brighter by virtue of their proximity, yielding much higher signal-to-noise. Moreover, some intrinsic differences make it possible to verify that relativistic $\mathrm{Fe} \mathrm{K}$ lines in Seyfert-1 galaxies are likely to be robust.

Low-energy curvature due to warm absorption complicates studies of relativistic emission lines in some Seyfert galaxies (e.g., NGC 3783). At least in the case of MCG-6$30-15$, rigorous work shows that the broad line is independent of low-energy curvature (e.g., Vaughan \& Fabian 2004; Youg et al. 2005). Low energy absorption is unimportant in stellar-mass black holes. For instance, the column density in O VII and O VIII disk wind absorption lines detected in GX 339-4 simultaneously with a relativistic Fe K emission line (Miller et al. 2004a) is 100 times lower than the column density detected in the same absorption lines in MCG-6-3015 (Lee et al. 2001), which is known to have moderate lowenergy absorption. More importantly, the low energy X-ray emission in stellar-mass black holes is typically dominated

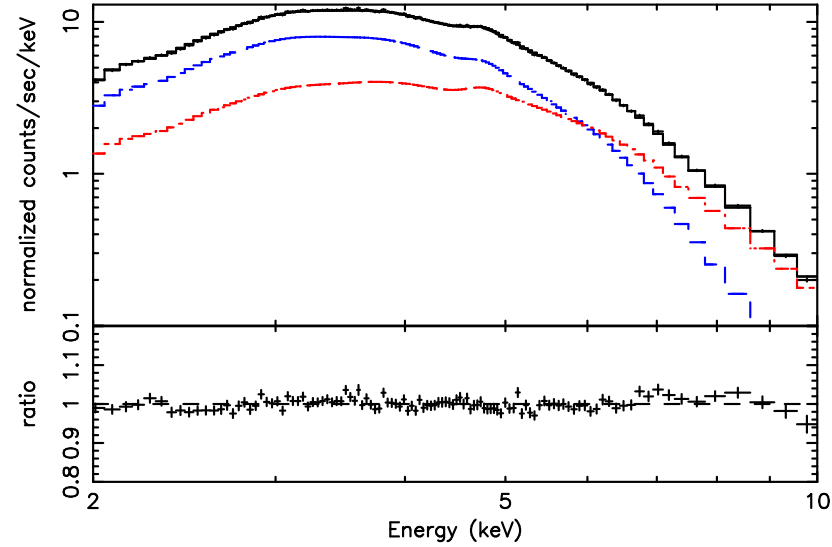

Fig. 2 An archival ASCA spectrum of the black hole candidate $4 \mathrm{U} 1630-472$ is shown above. The spectrum was fit with additive components, including a disk model (shown in blue) and a powerlaw model for the hard flux. The components clearly cross at $\sim$ $6 \mathrm{keV}$, but there is no indication for a broad $\mathrm{Fe} \mathrm{K}$ emission line in the data/model ratio. The need for two or more continuum flux components does not act to create false broad or relativistic Fe K emission lines in stellar-mass black hole spectra.

by thermal emission from the disk, which causes a spectral curvature that is the opposite of what absorption would cause.

It is sometimes argued that the multiple continuum flux components required to describe stellar-mass black holes might falsely create the need for a broad line. This concern is particularly important when additive components (e.g., from a phenomenological disk plus power-law model) cross near to the Fe K range. An archival ASCA spectrum of the black hole candidate $4 \mathrm{U} 1630-472$ is an excellent example that additive components do not easily create the need for an emission line (see Figure 2). When the spectrum is fitted with additive disk and power-law components that cross at about $6 \mathrm{keV}$, but there is no evidence for a broad emission line.

Given that relativistic lines can span $2-3 \mathrm{keV}$ in width at their base, accurate fits to the line (and, therefore, accurate spin constraints) depend on defining the underlying continuum spectrum well. It is possible to confuse this need with the idea that there is only one correct continuum model, and to worry that relativistic Fe $\mathrm{K}$ lines cannot be robust if different continuum models describe the continuum equally well. In spectra with strong Fe $\mathrm{K}$ lines and a well-defined (not perfectly defined) continuum, the line profile is largely independent of the continuum model assumed. Four fits to the continuum spectrum of GX 339-4 are shown in Figure 3 (Miller et al. 2006a), and the line profile is clearly robust against these differences. In spectra with a low signal-tonoise ratio, the choice of continuum model can affect the parameters one obtains when fitting a relativistic line profile, but this is not the case in good spectra.

Finally, it is sometimes argued that - whatever the true spectral continuum - partial covering of the central engine might act to falsely create the appearance of a broad iron 

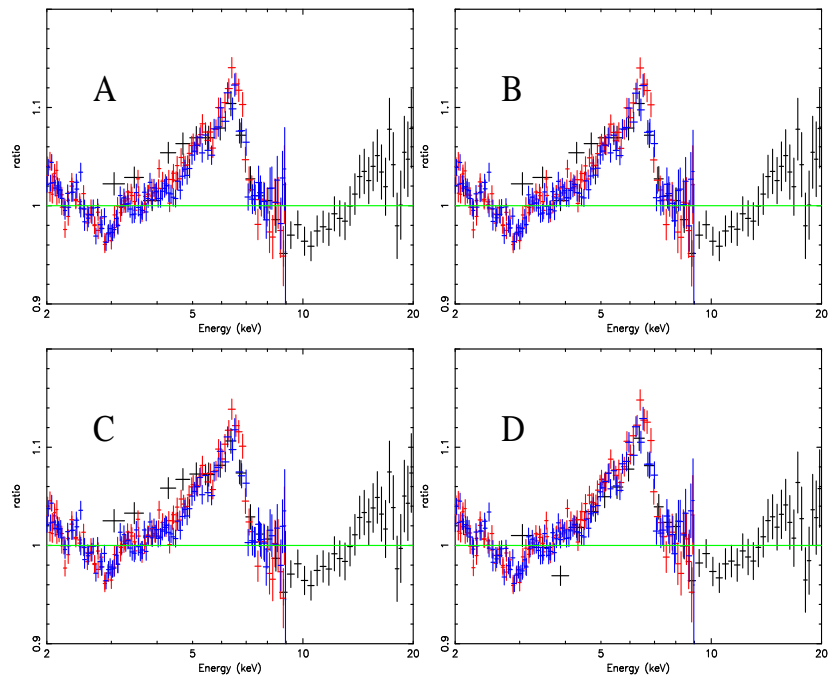

Fig. 3 The relativistic line profile revealed in simultaneous XMM-Newton (red and blue spectra) and RXTE (black spectra) observations of GX 339-4 is one among several that are robust against the particular choice of continuum model. Panel A shows the data/model ratio found when a simple "diskbb" plus powerlaw model is used to fit the data, panel $\mathrm{B}$ shows the ratio to a "diskpn" plus power-law model, panel C shows the ratio to a "diskbb" plus "CompTT" model, and panel D shows the ratio to the "bulk-motion Comptonization" model (see the text, and Miller et al. 2006a).

line by adding opacity in ionized K-shell absorption edges. The high signal-to-noise ratio achieved in long observations of stellar-mass black holes makes it possible to test this possibility. Figure 4 shows the XMM-Newton spectrum of GX 339-4 described in Miller et al. (2004b), fit with a disk plus power-law model, and allowing two edges to float in the Fe K edge band (7.1-9.3 keV) instead of adding a relativistic iron line and disk reflection. The spectrum is not well fitted by a model consistent with partial covering; indeed, the model is also significantly worse in a statistical sense. Partial covering also appears to be a significantly worse description of Seyfert-1 spectra where the high energy continuum is well-defined (Reynolds et al. 2004).

\section{Methods of Constraining Spin}

The frequency of high-frequency QPOs may be related to black hole spin, and most models for high-frequency QPOs point to high spin parameters, especially when the QPOs are seen in a 2:3 ratio. At present, however, a modulation mechanism has not been uniquely identified. In contrast, the mechanism by which relativistic iron lines are produced and shaped is well understood.

It is also possible to constrain the spin of a stellar-mass black hole by measuring the X-ray continuum flux from the accretion disk. In practice, this is complicated by many factors, including: the accuracy with which the ISM absorption is known, the assumed form of the hard component, the accuracy with which the distance and inclination of the system

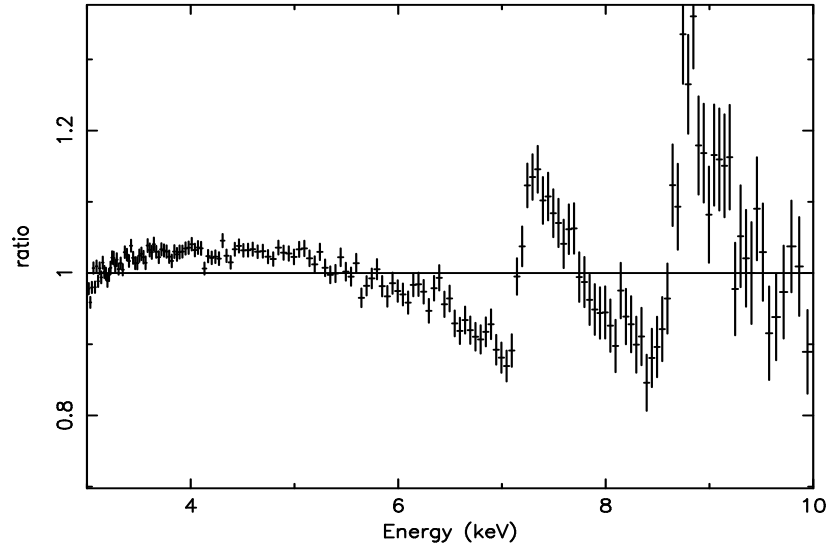

Fig. 4 The data/model ratio shown above is the result of fitting an absorbed "diskbb" plus power-law continuum to the $X M M$ Newton/EPIC-pn spectrum of GX 339-4 in a bright state. Two edges were allowed to float between $7.1-9.3 \mathrm{keV}$, as per a scenario in which patchy absorption partially covers the central engine. The resulting fit shown above is clearly unacceptable, and gives a significantly worse fit statistic than fits with a relativistic line model $\left(\chi^{2} / \nu=5913.5 / 1899\right.$, versus $\chi^{2} / \nu=3456.5 / 1894$, Miller et al. 2004b). In good spectra from stellar-mass black holes, partial covering can be ruled-out, further demonstrating that relativistic line profiles are robust.

and mass of the black hole are known, the assumed inner disk torque prescription, the mass accretion rate through the disk (which contains an uncertainty in the $\alpha$ viscosity parameter), and the accuracy with which spectral hardening through the disk atmosphere can be characterized.

As an example of the way in which estimates of black hole spin based on the disk continuum are subject to considerable systematic uncertainty, we consider an RXTE/PCA spectrum of the $4 \mathrm{U} 1543-475$ (observation 70133-01-0600, which started on 2002-06-29 at 14:08:16 TT). Shafee et al. (2006) previously fit the spectrum from this observation with a new disk model, having identified it as being a true soft state spectrum. 4U 1543-475 is a better choice for this test than, e.g., GRS 1915+105 (found to have a high spin parameter by McClintock et al. 2006 based on disk fits) as $4 \mathrm{U} 1543-475$ has a much lower column density, which considerably reduces one source of systematic difficulty in measuring the disk continuum.

We used the standard PCU spectrum available from the RXTE archive in the $2.8-25.0 \mathrm{keV}$ band. To further reduce a systematic uncertainty, the column density was fixed at $N_{H}=4.0 \times 10^{21} \mathrm{~cm}^{-2}$ as per Dickey \& Lockman (1990). We first fit this spectrum with a simple "diskbb" plus powerlaw model, and next with a "diskbb" plus "CompTT" model (with the initial temperature set to that of the disk, and the coronal temperature fixed to $50 \mathrm{keV}$ ). These models give statistically equivalent fits, and both models yield a disk color temperature of $k T=0.89 \mathrm{keV}$. However, they imply very different disk fluxes, and it is the flux that determines the emitting area and thereby the proximity of the disk to the black hole. Figure 5 shows that even when fitting spec- 


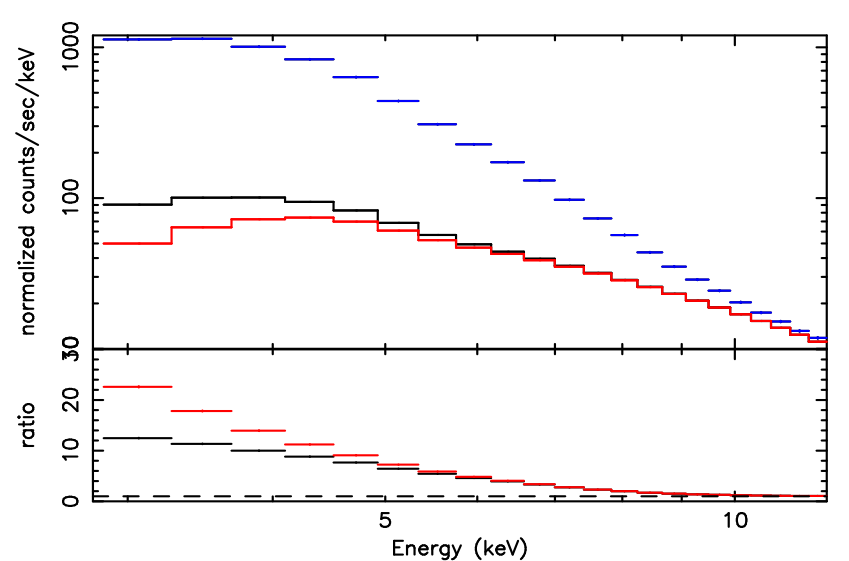

Fig. 5 Two statistically equivalent fits to a spectrum of $4 \mathrm{U}$ 1543-475 (shown in blue) are shown above (the disk flux was later set to zero to make this figure). A model consisting of "diskbb" and power-law components is shown in black, and a model consisting of "diskbb" and "CompTT" components is shown in red. Even when the hard flux is minimal, the choice of hard component can strongly affect the disk flux that is measured, and efforts to constrain the spin of the black hole via the disk continuum. The data/model ratio above shows that the disk flux at $3 \mathrm{keV}$ differs by a factor of $\sim 2$, due only to the different models used to fit the hard X-ray flux.

tra obtained in a soft disk-dominated state, different models for the hard component can generate a factor of 2 difference in the disk flux inferred at $3 \mathrm{keV}$.

We next fit the spectrum of 4U 1543-475 with the new variable-spin disk model considered in Shafee et al. (2006) and McClintock et al. (2006), using the same power-law model for the hard component. The "Kerrbb" model (Li et al. 2005) includes the spin of the black hole as a variable parameter, but requires one to know a torque/accretion power dissipation ratio, the inclination of the inner disk, the mass of the black hole, the mass accretion rate (which depends on the torque to accretion dissipation ratio), the distance to the black hole, the spectral hardening factor for transfer through the disk atmosphere, whether or not disk selfirradiation is important (a binary switch), and whether or not limb-darkening should be included (also a binary switch). In our fits, we assumed the default switches (a torque/accretion dissipation ratio of zero, inclusion of self-irradiation, and zero limb darkening), the mass of the black hole was constrained to $9.4 \pm 2.0 M_{\odot}$, its distance was constrained to $7.5 \pm 1.0 \mathrm{kpc}$, and the inclination was fixed to 20.7 degrees, as per Park et al. (2004). We find that fits with $a=0.9$ and $a=0.3$ are statistically equivalent for a small range in mass accretion rate. Clearly, constraints on the black hole spin parameter using the disk continuum are strongly affected by the choice of the hard component, and by the assumed parameters in the disk model.

In summary, relativistic iron lines are not the only way of constraining spin in stellar-mass black holes, but they present a few specific advantages over other methods. The examination in this and the previous section shows that lines

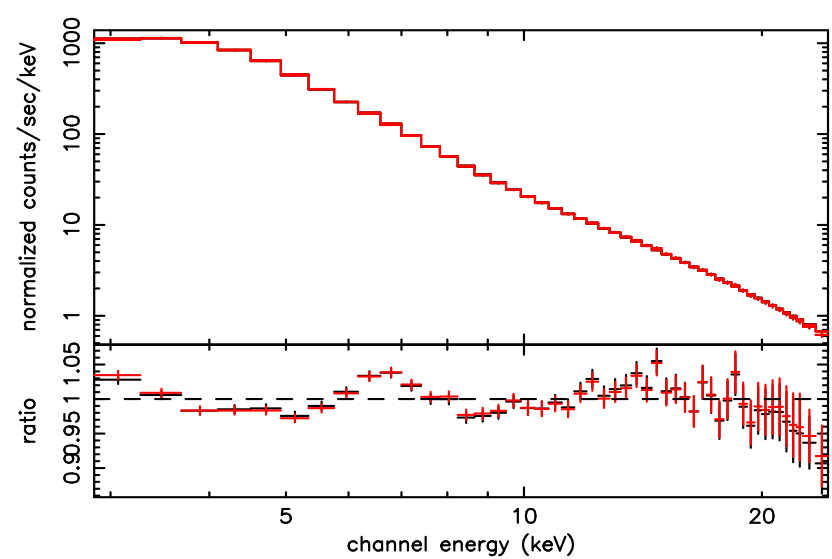

Fig. 6 Two examples of the same spectrum of 4U 1543-475 are shown above. Both examples are fit with a model including the "Kerrbb" disk and a power-law components. The model shown in black was fit with $a=0.9$ fixed, and the model in red was fit with $a=0.3$ fixed (please see the text). The data/model ratio clearly shows that both continuum fits are acceptable, and statistically equivalent. Indeed, the difficulties inherent in obtaining robust black hole spin constraints using the disk continuum as illustrated here motivated efforts to use Fe K emission lines and QPOs for this purpose.

are relatively free of systematic difficulties, at least when compared to QPO and disk continuum spin constraints. The largest source of uncertainty for relativistic iron lines is the extent to which Comptonization might broaden the line. It has been shown that for Comptonization to contribute significantly, however, the scattering region must be as optically thick as the disk itself (e.g., Reynolds \& Wilms 2000), which is unphysical. The inclination of the inner accretion disk is important for both relativistic iron lines and disk continua, but spin constraints based on iron lines do not require one to know the mass of a given black hole or its distance, nor do such constraints rely upon the many other parameters important for accurate disk continuum fits. Both disk line and disk continuum diagnostics assume that emission from within the innermost stable circular orbit is negligible, which is reasonable if the material in the plunging region is hot and optically-thin.

\section{Implications for AGN and Future Directions}

The high signal-to-noise across the broad X-ray bandpass afforded by the high flux observed from stellar-mass black holes permits robust fits to relativistic Fe K lines in medium and high-resolution X-ray spectra. Relativistic line studies can explore General Relativistic effects near to black holes, the spin evolution of stellar-mass black holes, and can bear on the role of black hole spin in launching relativistic jets. Beyond these considerations, stellar-mass black hole spectra are an excellent point of comparison for X-ray spectra obtained from Seyfert-1 AGN. Whereas the nature of the low and high energy continuum in Seyfert-1 X-ray spectra 
is sometimes ambiguous, and whereas partial covering of the central engine (which might cause the false appearance of a relativistic line) can be hard to exclude, in stellar-mass black holes it is possible to show that strong relativistic lines are robust against such effects (see above). The powerful combination of XMM-Newton and RXTE spectra has been instrumental in demonstrating the robustness of relativistic lines in stellar-mass black holes.

As noted above, the best relativistic spectra obtained from stellar-mass black holes and Seyfert-1 AGN can be fit using the same broad-band disk reflection model (Miller et al. 2004b). Such findings serve to indicate that the ionization of the inner accretion disk is the only major difference between the inner accretion flows. Given these results, and the robust and widespread nature of relativistic lines in stellarmass black holes, it is reasonable to expect that relativistic lines should be common in Seyfert-1 AGN. Low-energy absorption and poor sensitivity in the energy range above the Fe $\mathrm{K}$ band may hinder strong detections of relativistic lines in soft X-ray spectrometers, but if stellar-mass black holes can drive our expectations then such lines should be fairly common. There are already indications that improved broad-band sensitivity alone provides stronger detections of relativistic lines in Seyfert-1 AGN (see the contributions by Nandra, Reeves, and Markowitz in this proceedings).

The near-term future for relativistic line studies is very promising, and a few directions may be especially important to obtaining improved spin constraints, evidence of related relativistic phenomena, and the nature of orbits close to black holes.

At present, only two relativistic line models are commonly fit: the "diskline" model (Fabian et al. 1989) and the "Laor" model (Laor 1991). The former describes lines originating around a zero-spin Schwarzschild black hole, and the latter describes line emission originating around a maximalspin Kerr black hole. The vast majority of broad iron lines are better described by the Kerr model; this fact, and the simple FWHM obtained when line profiles are fit with a Gaussian function, are already a strong indications that most black holes may have high spin parameters. If one makes the approximation that the line profiles expected at high spin parameters do not differ very greatly, then one can associate the inner radius measured using the Laor model with the innermost stable circular orbit, and derive a more conservative estimate of the black hole spin parameter. Recently, models for relativistic lines with spin as a variable parameter have been calculated, and are available for spectral fitting in standard packages. These models are called "kdline" (Beckwith \& Done 2004, 2005) and "ky" (Dovciak, Karas, $\&$ Yaqoob 2004). A third such model (not yet public) was used to fit the line profile in MCG-6-30-15, and found a spin that is broadly consistent with some estimates using the Laor model ( $a \geq 0.987$, Brenneman \& Reynolds 2006). In the near future, it is vital that similar fits be made to a number of broad Fe K line profiles, both in Seyfert-1 AGN and stellar-mass black holes.
In AGN, robust QPOs have not yet been detected, and measuring the disk continuum is even more difficult than in stellar-mass black holes. The possibility of constraining spin via independent diagnostics, then, is unique to stellarmass black holes. Though each method of constraining spin has advantages and disadvantages, the best way forward for stellar-mass black holes will be to bring each diagnostic to bear simultaneously, and in as many sources as possible.

Studies of Fe K line and continuum variability are another important direction for the future. As noted above, a model including the effects of relativistic light bending close to a spinning black hole predicts a specific pattern of line and continuum variability (Miniutti et al. 2003). The variability patterns seen in some Seyfert-1 AGN and one stellar mass black hole appear to follow this pattern (Miniutti, Fabian, \& Miller 2004; Rossi et al. 2005). Until recently, most studies of Fe K lines in both Seyfert-1 AGN and stellar-mass black hole have focused on establishing the presence of relativistic lines in time-averaged spectra, and in measuring the parameters of the time-averaged line profile. Long observations already made with ASCA, BeppoSAX, Chandra, and XMM-Newton offer the possibility of further testing this model with Seyfert-1 AGN. In the case of stellar-mass black holes, long observations with these observatories, but also with RXTE, offer the same opportunity. Indeed, RXTE has obtained hundreds of observations of many stellar-mass black holes in outburst, but the line versus continuum flux has only been examined in one case so-far. Light bending should occur near to black holes, especially those with high spin parameters, and variability studies may be able to reveal this effect.

Finally, searches for periodic and quasi-periodic signatures in relativistic iron line profiles and disk reflection hold extraordinary promise. The orbital timescales in the inner disk around supermassive black holes can now be probed with XMM-Newton and Suzaku. Nearly periodic line variability consistent with a flare orbiting the black hole at 7$16 G M / c^{2}$ was detected in a long observation of NGC 3516 (Iwasawa, Miniutti, \& Fabian 2004). A similar periodicity has also been reported in long XMM-Newton observation of Mrk 766 (Turner et al. 2006). The statistical significance of these periods is modest, but future long observations can prove decisive. In stellar-mass black holes, the equivalent width and flux of $\mathrm{Fe} \mathrm{K}$ emission lines in the spectrum of GRS 1915+105 has recently been found to vary with QPO phase (Miller \& Homan 2005). This can be explained in terms of precession in the inner disk (Schnittman, Homan, \& Miller 2006), and may hint at Lense-Thirring precession. While it is seldom that a QPO is observed with the high rms amplitude required for such studies, this is again an area in which future observations with RXTE, XMM-Newton, and Suzaku can reveal more information on orbits close to black holes.

In summary, comparing and contrasting relativistic $\mathrm{Fe} \mathrm{K}$ lines and disk reflection in Seyfert-1 AGN and stellar-mass black holes is extremely valuable, and continues to yield 
important insights. There is a bright future for studies of lines in stellar-mass black holes using the present array of $\mathrm{X}$-ray observatories; significant progress is within reach. In the long run, the insights that relativistic lines in stellar-mass black hole spectra provide strongly argue in favor of retaining a bright (1-2 Crab) source capacity in future X-ray missions such as Constellation-X and XEUS.

Acknowledgements. I wish to acknowledge Chris Reynolds and Andy Fabian for comments on this contribution. I would like to thank Norbert Schartel, Andy Fabian, Arvind Parmar, Matthias Ehle, Maria Diaz-Trigo, and the XMM-Newton staff responsible for arranging an excellent conference.

\section{References}

Barr, P., White, N. E., \& Page, C. G.: 1985, MNRAS, 216, 65

Beckwith, K., \& Done, C., 2004, MNRAS, 352, 353

Beckwith, K., \& Done, C., 2005, MNRAS, 359, 1217

Brenneman, L. W., \& Reynolds, C. S.: 2006, ApJ, in press, astro$\mathrm{ph} / 0608502$

Diaz Trigo, M., Parmar, A. N., Miller, J. M., \& Kuulkers, E.: 2006, A\&A, in press

Dickey, J. M., \& Lockman, F. J.: 1990, ARA\&A, 28, 215

Dovciak, M., Karas, V., \& Yaqoob, T.: 2004, ApJS, 153, 205

Ebisawa, K., Ueda, Y., Inoue, H., Tanaka, Y., \& White, N. E.: 1996, ApJ, 467, 419

Fabian, A. C., Rees, M. J., Stella, L., \& White, N. E.: 1989, MNRAS, 238, 729

Fabian, A. C., Miniutti, G., Iwasawa, K., \& Ross, R. R.: 2005, MNRAS, 361, 795

George, I. M., \& Fabian, A. C.: 1991, MNRAS, 249, 352

Iwasawa, K., et al.: 1996, MNRAS, 282, 1038

Iwasawa, K., Miniutti, G., \& Fabian, A. C.: 2004, MNRAS, 355, 1073

Laor, A.: 1991, ApJ, 376, 90

Lee, J. C., et al.: 2001, ApJ, 554, L13

Li, L., Zimmerman, E. R., Narayan, R., \& McClintock, J. E., 2005, ApJS, 157, 335

Martocchia, A., Matt, G., Karas, V., Belloni, T., \& Feroci, M.: 2002, A\&A, 387, 215

Martocchia, A., Matt, G., Belloni, T., Feroci, M., Karas, V., \& Ponti, G.: 2006, A\&A, 448, 677

McClintock, J. E., Shafee, R., Narayan, R., Remillard, R., Davis, S. W., \& Li, L.: 2006, ApJ, in press, astro-ph/0606076

Miller, J. M., et al.: 2002a, ApJ, 578, 348

Miller, J. M., et al.: 2002b, ApJ, 570, L69

Miller, J. M., Fabian, A. C., in 't Zand, J. JM., Reynolds, C. S., Wijnands, R., Nowak, M. A., \& Lewin, W. H. G.: 2002c, ApJ, 577, L15

Miller, J. M., et al.: 2004a, ApJ, 601, 450

Miller, J. M., et al.: 2004b, ApJ, 606, L131

Miller, J. M., Fabian, A. C., Nowak, M. A., \& Lewin, W. H. G.: 2004, in the proceedings of the Tenth Marcel Grossmann Meeting, Rio de Janiero, 2003, eds. M. Novello, S. Perez Bergliaffa, R. Ruffini, Singapore: World Scientific Publishing

Miller, J. M., \& Homan, J.: 2005, ApJ, 618, L107

Miller, J. M., et al.: 2006a, ApJ, in press, astro-ph/0602633

Miller, J. M., et al.: 2006b, ApJ, 646, 394

Miniutti, G., Fabian, A. C., Goyder, R., \& Lasnby, A. N.: 2003, MNRAS, 344, L22

Miniutti, G., Fabian, A. C., \& Miller, J. M.: 2004, MNRAS, 351, 466

Nandra, K., Mushotzky, R. F., Yaqoob, T., George, I. M., \& Turner, T. J.: 1996, MNRAS, 284, L7

Nandra, K., George, I. M., Mushotzky, R. F., Turner, T. J., \& Yaqoob, T.: 1997, MNRAS, 477, 602

Park, S. Q., et al.: 2004, ApJ, 610, 378

Ponti, G., Miniutti, G., Cappi, M., Maraschi, L., Fabian, A. C., \& Iwasawa, K.: 2006, MNRAS, 368, 903

Reynolds, C. S., \& Wilms, J., 2000, ApJ, 533, 821

Reynolds, C. S., Wilms, J., Begelman, M. C., Staubert, R., \& Kendziorra, E.: 2004, MNRAS, 349, 1153

Rossi, S., Homan, J., Miller, J. M., \& Belloni, T.: 2005, MNRAS, 360,763

Schnittman, J. D., Homan, J., \& Miller, J. M.: 2006, ApJ, 642, 420 
Shafee, R., McClintock, J., Narayan, R., Davis, S. W., Li, L., \& Remillard, R. A.: 2006, ApJ, 636, L113

Strohmayer, T. E.: 2001, ApJ, 552, L49

Tanaka, Y., et al.: 1995, Nature, 375, 659

Turner, T., J., Miller, L., George, I. M., \& Reeves, J. N., 2006, A\& A, 445,59

Vaughan, S., \& Fabian, A. C.: 2004, MNRAS, 348, 1415

van der Woerd, H., White, N. E., \& Kahn, S. M.: 1989, ApJ, 344, 320

Young, A. J., Lee, J. C., Fabian, A. C., Reynolds, C. S., Gibson, R. R., \& Canizares, C. R.: 2005, ApJ, 631, 733

in 't Zand, J. J. M., et al.: 2002, A\&A, 390, 597

in 't Zand, J. M. M., Miller, J. M., Oosterboek, T., \& Parmar, A. N.: 2002, A\&A, 394, 553 